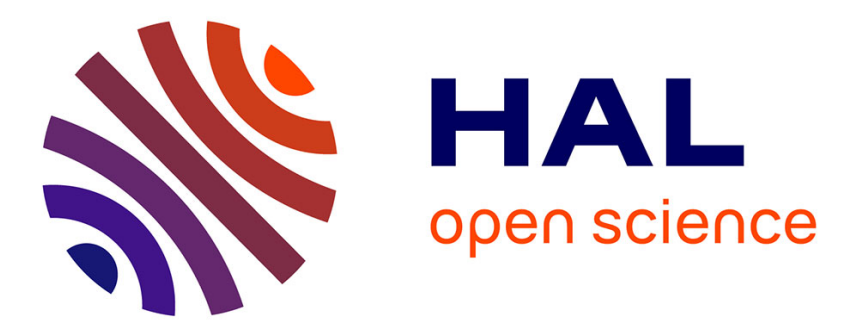

\title{
Rôle de l'impulsivité dans la relation entre surpoids et TDAH chez l'enfant : étude exploratoire des caractéristiques cliniques, neuropsychologiques et neurobiochimiques
}

\author{
L. Ben Amor, J. Lachal
}

\section{To cite this version:}

L. Ben Amor, J. Lachal. Rôle de l'impulsivité dans la relation entre surpoids et TDAH chez l'enfant: étude exploratoire des caractéristiques cliniques, neuropsychologiques et neurobiochimiques. L'Encéphale, 2019, 45, pp.494 - 500. 10.1016/j.encep.2019.06.002 · hal-03488425

\author{
HAL Id: hal-03488425 \\ https://hal.science/hal-03488425
}

Submitted on 21 Dec 2021

HAL is a multi-disciplinary open access archive for the deposit and dissemination of scientific research documents, whether they are published or not. The documents may come from teaching and research institutions in France or abroad, or from public or private research centers.
L'archive ouverte pluridisciplinaire HAL, est destinée au dépôt et à la diffusion de documents scientifiques de niveau recherche, publiés ou non, émanant des établissements d'enseignement et de recherche français ou étrangers, des laboratoires publics ou privés.

\section{(c) (1) $\$$}

Distributed under a Creative Commons Attribution - NonCommerciall 4.0 International 
Rôle de l'impulsivité dans la relation entre surpoids et TDAH chez l'enfant : étude exploratoire des

caractéristiques cliniques, neuropsychologiques et neurobiochimiques

Impulsivity and obesity in children with Attention Deficit Hyperactivity Disorder: a clinical, neuropsychological and magnetic resonance spectroscopy exploratory study

\section{Leila BEN AMOR ${ }^{1,2}$, Jonathan LACHAL $2,3,4,5 *$}

${ }^{1}$ Département de psychiatrie, CHU Sainte-Justine, 3175 Chemin de la Côte-Sainte-Catherine, Montréal, QC H3T 1C5, Canada

${ }^{2}$ Département de psychiatrie, Université de Montréal

${ }^{3}$ AP-HP, Cochin Hospital, Maison de Solenn, Paris, France

${ }^{4}$ Université Paris Descartes, Sorbonne Paris Cité, Paris, France

${ }^{5}$ CESP, Fac. de médecine - Univ. Paris-Sud, Fac. de médecine - UVSQ, INSERM, Université ParisSaclay, 94805, Villejuif, France

* Pour correspondance : Jonathan Lachal

AP-HP, Cochin Hospital, Maison de Solenn, Université Paris Descartes, CESP- INSERM 1178, PARIS, France

e-mail : jonathan.lachal@gmail.com 


\title{
Rôle de l'impulsivité dans la relation entre surpoids et TDAH chez l'enfant : étude exploratoire des caractéristiques cliniques, neuropsychologiques et neurobiochimiques
}

\author{
Impulsivity and obesity in children with Attention Deficit Hyperactivity \\ Disorder: a clinical, neuropsychological and magnetic resonance \\ spectroscopy exploratory study
}

\section{Résumé}

Objectifs: De nombreux résultats mettent en évidence les liens entre trouble déficit de l'attention/hyperactivité (TDAH) et obésité chez l'adulte et l'enfant, sans toutefois bien en comprendre la physiopathologie. Cette étude exploratoire a pour objectif de comparer un groupe d'enfants atteints d'un TDAH avec surpoids et des enfants atteints d'un TDAH de poids normal au niveau de leurs caractéristiques cliniques, neuropsychologiques et neurobiochimiques, en ciblant particulièrement les marqueurs de l'impulsivité. Méthode : Parmi des enfants souffrant de TDAH (5-12ans), dix enfants avec un surpoids ou obésité ont été appariés pour le sexe et l'âge à dix enfants de poids normal. Les Conners Rating Scales versions parents (CPRS) et professeur (CTRS) ainsi que le Conners' Continuous Performance Test II (CPT-II) ont été réalisés. Sur le plan neurobiochimique, les ratios des métabolites cérébraux (glycérophosphocholine + phosphocholine/créatinine $(\mathrm{GPC}+\mathrm{PCh} / \mathrm{Cr})$, glutamate + glutamine/Cr $(\mathrm{Glu}+\mathrm{Gln} / \mathrm{Cr})$, myoinositol/Cr $(\mathrm{mI} / \mathrm{Cr})$ et $\mathrm{N}$-acétylaspartate $+\mathrm{N}$-acétylaspartylglutamate/Cr (NAA+NAAG/Cr)) ont été mesurés par spectroscopie par résonance magnétique (SRM) dans les régions d'intérêt. Résultats : Les résultats aux échelles hyperactivité-impulsivité et Conners Global Index (CGI) du CTRS-R sont en faveur d'une plus grande impulsivité chez les enfants avec surpoids. Les deux groupes sont comparables pour les résultats neuropsychologiques. La $\mathrm{SRM}$ met en évidence un ratio $\mathrm{GPC}+\mathrm{PCh} / \mathrm{Cr}$ supérieur dans le cortex préfrontal droit des enfants présentant un surpoids. Conclusions : Les résultats renforcent l'hypothèse du lien entre obésité et impulsivité chez les enfants souffrant de TDAH, ainsi que l'implication des régions préfrontales droites.

Mots clés : TDAH, impulsivité, obésité, surpoids, spectroscopie.

\section{Abstract}

Objectives: Relationship between Attention Deficit Hyperactivity Disorder (ADHD) and obesity in adults and children had previously been established in research studies. Brain imaging studies pointed out the important role of the prefrontal region in both ADHD and obesity. However, the underlying link between ADHD and obesity is not well understood. The hypothesis that impulsivity could play a role has been explored in clinical studies of ADHD and Binge Eating Disorders or Loss of Control Eating, with contradictory results. Our study aims to compare 
children with ADHD and obesity to children with ADHD and normal weight. We propose to compare these two populations with clinical, neuropsychological and brain spectroscopy investigation, focusing specifically on impulsivity items. Method: Ten children presenting overweight or obesity were selected from a larger population of children with ADHD (5-12y) and paired with regard to gender and age with ten children with ADHD and normal weight from the same population. Conners Rating scales version parents (CPRS) and teachers (CTRS), Conners' Continuous Performance Test II (CPT-II), and Magnetic Resonance Spectroscopy (MRS) metabolites in five regions of interest (left and right prefrontal, left and right striatal and left cerebellum regions) were measured for all the children. For MRS, ratio to creatinine levels of following metabolites were measured: glycerophosphocholine + phosphocholine/creatinine $(\mathrm{GPC}+\mathrm{PCh} / \mathrm{Cr})$, glutamate + glutamine $(\mathrm{Glu}+\mathrm{Gln} / \mathrm{Cr})$, myoinositol $(\mathrm{mI} / \mathrm{Cr})$ et $\mathrm{N}$-acétylaspartate $+\mathrm{N}$-acétylaspartylglutamate (NAA+NAAG/Cr). Results: Hyperactivity/Impusivity and Conners Global Index (CGI) subscales of Conners rating scales showed a higher rate of impulsivity in children with ADHD and obesity as compared to children with ADHD and normal weight. Neuropsychological results were comparable in the two groups. Finally, MRS showed a higher $\mathrm{GPC}+\mathrm{PCh} / \mathrm{Cr}$ ratio in right prefrontal cortex in children with ADHD and obesity as compared to children with ADHD and normal weight. Conclusions: our results are concordant with the hypothesis that impulsivity could be the link between obesity and ADHD in a population of children with ADHD. The right prefrontal regions seem to be areas of interests that need more research in the study of the link between obesity and ADHD.

Keywords: ADHD, impulsivity, obesity, overweight, spectroscopy

\section{Introduction}

Le trouble déficit de l'attention/hyperactivité (TDAH) est un trouble neurodéveloppemental fréquent avec une prévalence mondiale se situant entre 3 et $10 \%$ [4]. Il est caractérisé par deux types d'atteinte, l'hyperactivité/impulsivité et le déficit attentionnel, le diagnostic reposant sur une atteinte cliniquement significative dans l'un ou les deux domaines [1]. Le TDAH a des conséquences sociétales majeures - impact psychologique, familial, scolaire et professionnel, et coût financier important $[2,3]$.

Le TDAH est fréquemment associé à de nombreuses comorbidités psychiatriques - Troubles oppositionnels et troubles des conduites, troubles de l'apprentissage et du langage, troubles de l'humeur, troubles anxieux, ou encore troubles du spectre autistique - et somatiques comme l'hypertension, le diabète, les troubles du sommeil, et l'obésité [4,5]. De nombreuses études mettent en évidence aujourd'hui les liens entre l'obésité, le surpoids, et le TDAH [3,4,6-8]. Des 
études transversales ont mis en évidence le lien statistique significatif de la co-occurrence du TDAH et de l'obésité et du surpoids chez l'adulte, mais aussi chez l'adolescent et l'enfant, ceci en population clinique et en population générale, et en contrôlant les multiples facteurs de confusion [8-10]. Des études longitudinales laissent apparaître le TDAH comme facteur de risque de l'obésité $[3,11]$. En résumé, la corrélation entre TDAH et obésité serait statistiquement significative, elle augmenterait avec l'âge de l'enfant, elle concernerait également le surpoids, et pourrait être directionnelle - le TDAH étant alors considéré comme facteur de risque d'obésité $[5,6]$.

Ce lien a des conséquences majeures en termes de santé publique [9,12]. L'obésité ne cesse d'augmenter en Amérique du Nord et à travers le monde avec une prévalence de 18,5\% aux USA en 2016 [13], avec des conséquences importantes sur la morbidité et mortalité à long terme [14,15]. Chez les enfants, le double diagnostic de TDAH et d'obésité engendre des conséquences psychologiques importantes: stigmatisation sociale, baisse de l'estime de soi et difficultés scolaires [16].

Pourtant, on connaît encore mal les mécanismes physiopathologiques qui sous-tendent ces liens [4]. De nombreuses hypothèses ont été formulées, mettant en jeu l'impulsivité motrice (en lien avec l'impulsivité alimentaire), les dysfonctionnements dopaminergiques (hypothèse du déficit de l'axe de la récompense), l'inattention (diminution de la réceptivité aux signaux de satiété, peu de régulation et de planification alimentaire), des mécanismes neurobiologiques et génétiques communs, potentiellement modulés par la présence d'affects dépressifs, ou les troubles du sommeil $[3,5,7,9]$. Le traitement précoce par psychostimulant serait quant à lui un moyen de prévenir la prise de poids associée au TDAH - effet anorexigène des traitements psychostimulant et diminution des symptômes de TDAH qui favorisent la prise de poids. 
L'hypothèse de l'impulsivité a été explorée à l'aide de différents paradigmes. L'impulsivité, et en particulier le déficit d'inhibition de la réponse et la difficulté à tolérer les délais seraient liés à des comportements alimentaires anormaux qui engendrent l'obésité [17]. Les dysfonctionnements cérébraux dans le système de la récompense ont d'ailleurs été décrits à la fois dans le TDAH et dans l'obésité [18].

D'autre part, l'impulsivité motrice du TDAH et l'impulsivité alimentaires pourraient être similaires. Cependant, les études récentes ont du mal à mettre en évidence un lien entre l'impulsivité mesurée du TDAH et les comportements de type accès hyperphagiques (Binge Eating Disorders - BED), ou Perte du contrôle alimentaire (Loss of Control Eating - LOC) [4,9,10,19]. Le TDAH pourrait être associé à la part objective du BED, mais pas à sa part subjective [4]. Plus encore, l'impulsivité mesurée par les échelles dans le TDAH ne serait pas du même type que l'impulsivité alimentaire présente dans le BED et le LOC [9]. Certains auteurs suggèrent d'étudier l'effet des différents sous-types de TDAH [6].

Enfin, l'impulsivité est associée à une moindre activité physique elle-même reliée au surpoids et à l'obésité [20].

Les études neuropsychologiques sont contrastées. Les enfants les plus impulsifs aux Door Opening Task, Stop Signal Task et Matching Familiar Figure Test (MFFT) ou encore au DelisKaplan Executive Function System sont ceux qui présentent l'Indice de Masse Corporelle (IMC) le plus important [21]. Mais ces résultats n'ont pu être retrouvés dans les tests utilisant le Continuous Performance Test II (CPT-II) [22].

Au niveau neurofonctionnel, les études récentes montrent une superposition de la carte des circuits neurocomportementaux impliqués dans le TDAH et ceux impliqués dans l'obésité [10]. Les études en neuroimagerie fonctionnelle soutiennent l'implication des régions préfrontales, 
striatales et cérébelleuses dans le TDAH [23]. En particulier, l'aire frontale (gyrus frontal supérieur, gyrus frontal précentral et inférieur), l'aire pariétale (gyrus post-central, gyrus angulaire, sillon sous-pariétal), temporale (gyrus temporal supérieur) et occipital (gyrus occipital médian), le cortex cingulaire, l'insula, et les noyaux de la base sont impliqués chez l'enfant [24]. Les études portant sur les sous-groupes d'enfants présentant un TDAH et des troubles caractérisés par une alimentation excessive (obésité, BED, LOC) montrent des anomalies communes aux deux troubles, portant plus précisément sur les circuits de la récompense (ganglions de la base, amygdale, cortex orbito-frontal et préfrontal), de l'inhibition de réponse (circuits fronto-striataux et fronto-cérébelleux) et de la régulation et du traitement des émotions (amygdale, insula, striatum, cortex cingulaire antérieur, cortex préfrontal) [10].

Enfin, l'utilisation de la spectroscopie par résonance magnétique chez les enfants souffrant de TDAH a montré des anomalies de ratio de métabolites cérébraux dans la boucle cortico-striatale et dans le cervelet (anomalies du ratio entre choline, myoinotisol, N-acétylaspartate ou glutamate-glutamine d'une part et créatinine d'autre part) [25-27]. Si aucune étude n'a été publiée avec une technique similaire chez les enfants souffrant d'obésité, les recouvrements neuroanatomiques et neurofonctionnels de l'obésité et du TDAH permettent d'envisager l'hypothèse de mécanismes neurométaboliques communs. Mais il est nécessaire de proposer des études comparatives examinant chaque facteur séparément et en association. À notre connaissance, aucune étude de ce type n'a encore été publiée.

L'objectif principal de cette étude est donc de comparer un groupe d'enfants atteints d'un TDAH avec surpoids et des enfants atteints d'un TDAH de poids normal au niveau de leurs caractéristiques cliniques, neuropsychologiques et neurobiochimiques. Nous posons l'hypothèse que l'impulsivité sera plus marquée chez les enfants ayant un surpoids que chez les enfants de poids normal, et qu'ils présenteront des différences au niveau de l'activité métabolique cérébrale. 
En particulier, nous proposons d'explorer les anomalies précédemment identifiées dans le TDAH et qui correspondent à des aires de perturbations neurofonctionnelles identifiées dans l'obésité : le cortex préfrontal, le striatum, et le cervelet. Nous posons l'hypothèse d'une activité cérébrale plus élevée ou plus basse identifiée par des ratios de métabolites plus élevés ou plus faibles dans ces aires cérébrales impliquées dans le processus de récompense et d'inhibition de la réponse. Pour vérifier cette hypothèse, nous proposons une étude analytique de type cas témoins avec appariement un pour un.

\section{Méthode}

\section{Population et Échantillonnage}

Les sujets ont été sélectionnés parmi les enfants ayant participé à une large étude visant à étudier l'imagerie génétique chez les enfants franco-canadiens présentant un TDAH (Imagerie génétique et TDAH chez les enfants franco-canadiens, sous la direction du Dr L Ben Amor, [28]). Ils ont été recrutés à la clinique externe de pédopsychiatrie de l'Hôtel-Dieu-de-Lévis (Québec, Canada) de 2005 à 2011.

Les critères d'inclusion étaient les suivants : (1) âgé de 5-12 ans ; (2) avoir reçu un diagnostic clinique de TDAH (diagnostic clinique établi par un pédopsychiatre selon les critères du DSMIV-TR [29], le pédopsychiatre disposait des résultats des mesures cliniques au moment de l'évaluation diagnostique).

Les critères de non-inclusion étaient : (1) traitement psychotrope avant l'évaluation initiale par le pédopsychiatre; (2) diagnostic clinique de trouble envahissant du développement (TED) ou de psychose ; (3) un QI $\leq 70$ (mesuré avec la Wechsler Intelligence Scale for Children, quatrième édition [30] pour les enfants de 6 ans et plus, ou la Wechsler Preschool and Primary Scale of Intelligence, troisième édition [31] pour les enfants de 5 ans). 
Parmi l'ensemble des sujets évalués, les sujets remplissant les critères d'inclusion et dont les données de taille et de poids au moment du diagnostic étaient disponibles ont été sélectionnés. L'IMC a été calculé, et les sujets ont été classés en deux groupes selon leur répartition par rapport au 85e rang centile (de Onis, 2007). Au total, dix sujets, dits cas, présentaient un surpoids selon cette définition, formant le groupe TDAH-surpoids (TDAH-SP).

Chacun de ces cas a été apparié individuellement par précision avec dix témoins de poids normal $\left(\geq 3^{\mathrm{e}}\right.$ et $\leq 85^{\mathrm{e}}$ rang centile) selon (1) leur genre et (2) leur âge, formant le groupe TDAH-poids normal (TDAH-PN). L'appariement n'a pas tenu compte du sous-type diagnostique de TDAH.

Le projet a été approuvé par le Comité d'éthique de l'Hôtel-Dieu-de-Lévis. Un consentement écrit a été obtenu de la part des parents pour l'évaluation de leur enfant.

\section{$\underline{\text { Recueil des données }}$}

Chaque sujet était évalué en clinique, à l'aide de tests neuropsychologiques et bénéficiait d'une spectroscopie par résonance magnétique.

\section{Mesures cliniques}

Les parents remplissaient un questionnaire sur les antécédents développementaux, médicaux, psychiatriques et familiaux de leurs enfants ainsi que des éléments sociodémographiques.

Les parents et les enseignants remplissaient les échelles de Conners révisées versions parents (CPRS-R) et professeur (CTRS-R) [32]. Ces échelles évaluent la perception qu'ont les parents et les enseignants des comportements de l'enfant. Le CPRS-R et le CTRS-R comptent diverses sous-échelles dérivées d'analyses factorielles. Les sous-échelles suivantes ont été retenues comme variables d'intérêt : (1) DSM-IV inattention, (2) DSM-IV hyperactivité-impulsivité, (3) Conners Global Index-Total (CGI-Total) et (4) DSM-IV total. Les sous-échelles DSM-IV inattention, hyperactivité-impulsivité et total réfèrent directement aux critères diagnostiques du 
TDAH dans le DSM-IV. L'échelle CGI-T est une mesure de psychopathologie globale [33]. Les résultats de ces sous-échelles sont rapportés sous forme de scores T. Les scores s'étendent de 0 à 100, avec une moyenne de 50 et un écart-type de 10, et des scores supérieurs à 65 sont considérés comme étant cliniquement significatifs [32]. Le CPRS-R et le CTRS-R sont des échelles qui sont à la fois sensibles et spécifiques pour prédire l'inattention et l'hyperactivité [32].

\section{Mesures neuropsychologiques}

Les participants complétaient le Continuous Performance Test II (CPT-II) [34]. Le test était administré par un assistant de recherche expérimenté et les résultats étaient vérifiés et discutés avec un neuropsychologue. Le diagnostic clinique n'était pas connu des évaluateurs. Les enfants pour qui un traitement avait été débuté après l'évaluation clinique devaient l'interrompre 24 heures avant l'épreuve. Les variables d'intérêt suivantes ont été retenues : (1) les omissions, (2) les commissions, (3) le temps de réaction, (4) l'erreur standard du temps de réaction, (5) la variabilité et (6) les persévérations. Les résultats du CPT-II sont rapportés sous forme de scores T. Les scores s'étendent de 0 à 100, avec une moyenne de 50 et un écart-type de 10.

\section{Mesures neurobiochimiques}

Tous les patients bénéficiaient d'une Spectrométrie par résonance magnétique du proton (SRM$1 \mathrm{H})$, réalisée avec un spectromètre-imageur Signa Horizon 1,5 T de GE opérant à $63,85 \mathrm{mHz}$ (General Electric Medical Systems, Waukesha, WI). Pour les participants sous traitement après l'évaluation clinique initiale, celui-ci était suspendu le matin de la SRM.

La localisation des voxels était déterminée par des séquences d’imagerie rapide, effectuées par l'acquisition d'images de tranches axiales en utilisant une séquence pondérée en T1 de spin-écho rapide. Les spectres étaient acquis dans des voxels de $8,00 \mathrm{~cm}^{3}$ localisés dans 5 régions d'intérêt (ROIs) : les régions préfrontales droite et gauche, striatales droite et gauche et cérébelleuse 
gauche. Pour la localisation des voxels, des paramètres anatomiques prédéterminés étaient utilisés (soit la portion postérieure de la tête du noyau caudé selon l'axe antéro-postérieur pour les aires striatales ; au-dessus de celles déterminant les voxels striataux pour les aires préfrontales ; et approximativement $3 \mathrm{~mm}$ latéralement au bord du quatrième ventricule pour l'aire cérébelleuse). Après la localisation de chacune des ROIs, le protocole GE PROBE, qui comporte une suppression du signal d'eau par la séquence CHESS, était appliqué, et suivi d'une acquisition de type PRESS (temps d'écho de $30 \mathrm{~ms}$, temps de répétition de $1500 \mathrm{msec}$, nombre d'acquisitions de 128, largeur spectrale de $2000 \mathrm{~Hz}$, nombre de points de 1024 et temps d'acquisition total de 40 minutes approximativement). Avec ces conditions d'acquisition, le ratio signal/bruit était de 12 ou plus pour les signaux principaux, s'étendant de 12 à 28 , selon les régions. Les spectres obtenus étaient analysés avec le logiciel LCModel version 6,0 (Provencher, 1993) et les ratios suivants étaient calculés pour chacune des 5 ROIs : glycérophosphocholine + phosphocholine/créatinine $(\mathrm{GPC}+\mathrm{PCh} / \mathrm{Cr})$, glutamate + glutamine/Cr $\quad(\mathrm{Glu}+\mathrm{Gln} / \mathrm{Cr})$, myoinositol/Cr (mI/Cr) et $\mathrm{N}$-acétylaspartate $+\mathrm{N}$-acétylaspartylglutamate/Cr (NAA+NAAG/Cr).

\section{Analyses statistiques}

Des tests de $\mathrm{Khi}^{2}$ ont été effectués pour comparer les groupes selon leur sous-type de TDAH. Afin de tester la qualité de l'appariement, des tests de McNemar ont été effectués pour déterminer si les paires cas témoins sont concordantes ou discordantes pour des facteurs confondants potentiels.

Des tests des rangs signés de Wilcoxon ont été effectués pour comparer les deux groupes au plan de l'âge, des variables comportementales (CPRS-R, CTRS-R), des variables neuropsychologiques (CPT-II) et des variables neurobiochimiques (ratios $\mathrm{GPC}+\mathrm{PCh} / \mathrm{Cr}$, $\mathrm{Glu}+\mathrm{G} \ln / \mathrm{Cr}, \mathrm{mI} / \mathrm{Cr}$ et NAA+NAAG/Cr). Pour certains participants, des données étaient 
manquantes. Les analyses ont été faites avec les données disponibles. Des tests bilatéraux ont été réalisés pour toutes les variables. Le niveau de significativité a été établi à 0.05 .

\section{Résultats}

\section{Description de la population}

L'appariement des cas et des témoins a permis d'obtenir des paires de sujets de sexe et d'âge comparables (Tableau 1). Les analyses n’ont révélé aucune différence significative entre les deux groupes pour le revenu familial des sujets. Les comorbités (trouble d'apprentissage, trouble d'opposition avec provocation et trouble anxieux) sont réparties inégalement dans les groupes. Finalement, 7 sujets ont un surpoids, 3 présentent une obésité (groupe TDAH-SP), quand les 10 sujets du groupe TDAH-PN ont un poids normal.

\section{$\underline{\text { Variables cliniques }}$}

La répartition des paires de sujets selon le sous-type clinique de TDAH est présentée dans le tableau 1. Les résultats aux échelles de Conners réalisées pour 18 participants - données obtenues pour 7 patients présentant un surpoids, 2 une obésité et 9 un poids normal - sont statistiquement significatifs pour la CTRS-R (professeurs) pour la variable impulsivité et CGITotal dans le groupe surpoids (Tableau 2).

\section{Variables neuropsychologiques}

Les variables d'intérêt au CPT-II réalisées pour 18 participants - même description que précédemment - sont comparables dans les deux groupes, excepté un nombre significativement moins élevé de persévérations chez les sujets ayant un surpoids $(T=-18,50 ; \mathrm{p}=0,03)$ (Tableau 3).

\section{Variables neurobiochimiques}

12 participants ont participé aux mesures spectrométriques -6 patients en surpoids et 6 contrôles de poids normal. Les résultats obtenus montrent une différence statistiquement significative entre 
les groupes pour le ratio $\mathrm{GPC}+\mathrm{PCh} / \mathrm{Cr}$ dans le cortex préfrontal droit, qui est plus élevé dans le groupe TDAH-SP $(\mathrm{T}=10,50 ; \mathrm{p}=0,03)$ (Tableau 4).

\section{Discussion}

Afin de mieux comprendre la relation entre le TDAH et le surpoids chez les enfants, notre étude exploratoire propose de comparer un groupe d'enfants atteints d'un TDAH ayant un surpoids avec des enfants atteints d'un TDAH de poids normal au niveau de leurs caractéristiques cliniques, neuropsychologiques et neurobiochimiques. Bien que la validation statistique de nos résultats soit limitée par le faible échantillon, ils permettent de dégager des hypothèses qui pourront être confirmées dans de prochaines études.

Nous avions posé hypothèse initiale que l'impulsivité serait plus marquée chez les enfants ayant un surpoids que chez les enfants de poids normal. Certains éléments vont en faveur de cette hypothèse. Les diagnostics cliniques émis par le pédopsychiatre, les TDAH de sous-types mixte et hyperactif-impulsif étaient significativement plus fréquents dans le groupe avec un surpoids au diagnostic clinique par le pédopsychiatre, après appariement à l'aveugle pour le sous-type de TDAH. L'impulsivité plus importante du groupe ayant un surpoids était aussi rapportée dans les échelles cliniques complétées par les enseignants. Enfin, les résultats neurobiochimiques montrent des niveaux significativement plus élevés de $\mathrm{GPC}+\mathrm{PCh} / \mathrm{Cr}$ dans le lobe frontal droit des enfants ayant un TDAH de type hyperactif-impulsif associé à un surpoids.

L'hypothèse de l'impulsivité comme médiateur entre le TDAH et l'obésité est explorée depuis plusieurs années [19,35]. Plusieurs études mettent en évidence un lien statistique positif entre impulsivité et prise de poids [36]. L'impulsivité pourrait contribuer à l'obésité par deux voies : en modifiant la quantité d'aliments ingérés (BED), ou le type d'aliment ingéré (aliments très caloriques) [37]. Dans notre étude, les variables Impulsivité et CGI-Total étaient significativement plus élevées chez les patients en surpoids pour les échelles de Conners version 
professeurs, ce qui renforce l'hypothèse de l'impulsivité. Les résultats de la version parents montrent la même tendance même si les résultats ne sont pas significatifs. Cependant, il est montré dans la littérature que les parents sont généralement des observateurs moins objectifs que les enseignants [38].

Des études récentes ont proposé l'hypothèse d'un lien entre impulsivité du TDAH et impulsivité alimentaire, notamment autour des diagnostics de BED et de LOC eating [4,9,10,19]. Ces études donnent des résultats souvent contrastés. Si le lien entre suralimentation, obésité, et impulsivité existe [39], il semble difficilement mesurable à l'aide des tests neuropsychologiques classiquement utilisés dans le TDAH [9].

Dans le CPT-II, l'impulsivité est habituellement marquée par un nombre élevé d'erreurs de commission [40], un long temps de réaction et une plus grande variabilité [41]. Nos résultats n'ont pas montré de différences significatives entre les deux groupes pour ces variables d'intérêt. Choudhry et coll. (2013) ont utilisé une batterie complète de tests neuropsychologiques, incluant le CPT-II, pour étudier le lien entre le TDAH et l'obésité [22]. Leurs conclusions montrent que les enfants atteints d'un TDAH de poids normal ont un profil cognitif comparable à ceux atteints d'un surpoids. Seules les caractéristiques socio-économiques étaient associées au surpoids. Par ailleurs, la corrélation entre les paramètres du CPT-II et les mesures cliniques d'hyperactivitéimpulsivité et d'inattention est variable $[42,43]$.

Matheson et coll. (2018) suggèrent de séparer les différentes facettes de l'impulsivité dans les études, notamment l'impulsivité attentionnelle et l'impulsivité motrice [19] qui pourraient être reliées différemment à l'impulsivité alimentaire. Notre étude ne comprenait malheureusement pas de mesures relatives aux comportements alimentaires, ce qui ne nous permet pas de conclure sur cette hypothèse. 
Enfin, récemment, Egbert et coll. (2018) mettent en évidence un lien entre TDAH et BED objectif, mais également avec l'hyperphagie simple (Objective Overeating, hyperphagie sans mécanisme impulsif apparent) [4]. Cela remet en question le lien direct entre impulsivité motrice et alimentaire. D’autres études sont nécessaires pour explorer cette hypothèse.

Sur le plan neurobiochimique, Une différence significative a été observée dans le cortex préfrontal droit, où le ratio $\mathrm{GPC}+\mathrm{PCh} / \mathrm{Cr}$ est apparu significativement plus élevé pour les sujets ayant un surpoids. Les composés de la choline sont les principales composantes de la membrane cellulaire. La signification de l'élévation du ratio $\mathrm{GPC}+\mathrm{PCh} / \mathrm{Cr}$ est encore mal connue, mais elle est le plus souvent associée à une dégradation cellulaire rapide pouvant être le témoin d'une pathologie dégénérative (Alzheimer, Sclérose multiples) ou d'une plus grande activité cérébrale [25,44,45]. Les régions préfrontales, impliquées dans les fonctions exécutives, sont fréquemment impliquées dans les atteintes caractérisant le TDAH. En imagerie par résonance magnétique fonctionnelle (fMRI), selon les tâches utilisées, on retrouve une hypoactivation ou une hypertactivation des régions préfrontales (gyrus frontal inférieur, supérieur et médian, cortex préfrontal inférieur, ventrolatéral, orbitofrontal) associées aux taches inhibitrices et à la stimulation du processus de récompense [10,24,46]. Des différences d'activation des régions préfrontales sont également décrites chez les enfants souffrant d'obésité, de manière plus inconstante suivant les moments étudiés - pré prandial précoce, post prandial [10]. L’implication de ces régions pourrait être associée à un déficit des fonctions exécutives dites «chaudes »régulation émotionnelle en situation de vie réelle, par opposition aux fonctions exécutives dites “froides" en situation de laboratoires - [7,47]. Si les résultats en SMR sont plus rares et plus inconstants, les changements les plus notables chez l'enfant souffrant de TDAH concernent les composants de la choline dans le striatum gauche et le lobe frontal droit [24,25,48]. Les anomalies observées dans notre étude vont donc dans le sens d'une confirmation au niveau 
neurométabolique des liens neuroanatomiques fonctionnels précédemment observés entre obésité et TDAH chez l'enfant.

Davantage d'études sont nécessaires, pour confirmer l'importance des régions préfrontales droites chez les sujets souffrant de TDAH et d'obésité comorbides. Les études couplées en électro-encéphalographie (EEG) et imagerie fonctionnelle pourraient aider à confirmer ces premiers résultats [24].

Notre étude est une étude exploratoire combinant des données cliniques, neuropsychologiques et neurobiochimiques chez des enfants souffrant de TDAH. Nos résultats permettent d'explorer finement la relation entre le surpoids et l'impulsivité. L'effet confondant des psychostimulants a été contrôlé et incluant des enfants qui n'avaient jamais pris de médication avant l'évaluation initiale.

Notre étude présente des limites. De part sa nature exploratoire, l'échantillon inclus est de petite taille. Cependant, l'appariement un-pour-un des sujets a permis de comparer précisément des individus de même sexe et âge, et de limiter l'impact de la taille de l'échantillon sur la puissance statistique. Nous n'avons pas effectué de corrections pour comparaisons multiples. Ce choix était également justifié par le fait que les variables des tests utilisés sont fortement corrélées entre elles. Le petit échantillon n'a pas permis de différencier des groupes surpoids et obésité. Enfin, le sous-type diagnostic déterminé par le psychiatre est en partie lié aux résultats des échelles de Conners qu'il avait en sa possession au moment du diagnostic. Cependant, ce diagnostic dépend de nombreux autres facteurs cliniques.

En conclusion, notre étude exploratoire a permis une meilleure caractérisation des enfants atteints de TDAH et de surpoids comorbides. Si le petit échantillon ne nous permet pas de conclure, des résultats significatifs ont été obtenus concernant les liens avec l'impulsivité aux 
échelles cliniques, et l'implication du cortex préfrontal droit en imagerie fonctionnelle spectroscopique. Ces résultats demandent à être répliqués dans de futures études.

\section{Remerciements}

Les auteurs aimeraient remercier le département de psychiatrie et de neurosciences de l’Université Laval pour le soutien aux résidents en psychiatrie.

\section{Conflits d'intérêts}

Aucun

\section{Références}

[1] American Psychiatric Association. Diagnostic and Statistical Manual of Mental Disorders, Fifth Edition (DSM-5®). American Psychiatric Pub; 2013.

[2] Chen Q, Kuja-Halkola R, Sjölander A, et al. Shared familial risk factors between attentiondeficit/hyperactivity disorder and overweight/obesity - a population-based familial coaggregation study in Sweden. J Child Psychol Psychiatry 2017;58:711-8.

[3] Cortese S, Tessari L. Attention-Deficit/Hyperactivity Disorder (ADHD) and Obesity: Update 2016. Curr Psychiatry Rep 2017;19.

[4] Egbert AH, Wilfley DE, Eddy KT, et al. Attention-Deficit/Hyperactivity Disorder Symptoms Are Associated with Overeating with and without Loss of Control in Youth with Overweight/Obesity. Child Obes 2018;14:50-7.

[5] Nigg JT, Johnstone JM, Musser ED, et al. Attention-deficit/hyperactivity disorder (ADHD) and being overweight/obesity: New data and meta-analysis. Clin Psychol Rev 2016;43:6779.

[6] Cortese S, Moreira-Maia CR, St. Fleur D, et al. Association Between ADHD and Obesity: A Systematic Review and Meta-Analysis. Am J Psychiatry 2016;173:34-43.

[7] Hanć T, Cortese S. Attention deficit/hyperactivity-disorder and obesity: A review and model of current hypotheses explaining their comorbidity. Neurosci Biobehav Rev 2018;92:16-28.

[8] Quesada D, Ahmed NU, Fennie KP, et al. AÀ Review: Associations Between Attentiondeficit/hyperactivity Disorder, Physical Activity, Medication Use, Eating Behaviors and Obesity in Children and Adolescents. Arch Psychiatr Nurs 2018;32:495-504.

[9] Martínez de Velasco R, Barbudo E, Pérez-Templado J, et al. Review of the association between obesity and ADHD. Actas Esp Psiquiatr 2015;43:16-23. 
[10] Seymour KE, Reinblatt SP, Benson L, Carnell S. Overlapping neurobehavioral circuits in ADHD, obesity, and binge eating: evidence from neuroimaging research. CNS Spectr 2015;20:401-11.

[11] Khalife N, Kantomaa M, Glover V, et al. Childhood Attention-Deficit/Hyperactivity Disorder Symptoms Are Risk Factors for Obesity and Physical Inactivity in Adolescence. J Am Acad Child Adolesc Psychiatry 2014;53:425-36.

[12] Kooij JJS. ADHD and Obesity. Am J Psychiatry 2016;173:1-2.

[13] Hales CM, Carroll MD, Fryar CD, Ogden CL. Prevalence of Obesity Among Adults and Youth: United States, 2015-2016. NCHS Data Brief 2017:1-8.

[14] Reilly JJ, Kelly J. Long-term impact of overweight and obesity in childhood and adolescence on morbidity and premature mortality in adulthood: systematic review. Int $\mathrm{J}$ Obes 2005 2011:891-8.

[15] Scott KM, Bruffaerts R, Simon GE, et al. Obesity and mental disorders in the general population: results from the world mental health surveys. Int J Obes 2007;32:192-200.

[16] Waring ME, Lapane KL. Overweight in children and adolescents in relation to attentiondeficit/hyperactivity disorder: results from a national sample. Pediatrics 2008;122:e1-6.

[17] Cortese S, Angriman M, Maffeis C, et al. Attention-deficit/hyperactivity disorder (ADHD) and obesity: a systematic review of the literature. Crit Rev Food Sci Nutr 2008;48:524-37.

[18] Cortese S, Vincenzi B. Obesity and ADHD: Clinical and Neurobiological Implications. In: Stanford C, Tannock R, editors. Behav. Neurosci. Atten. Deficit Hyperact. Disord. Its Treat., vol. 9, Berlin, Heidelberg: Springer Berlin Heidelberg; 2011, p. 199-218.

[19] Matheson BE, Eichen DM. A Review of Childhood Behavioral Problems and Disorders in the Development of Obesity: Attention Deficit/Hyperactivity Disorder, Autism Spectrum Disorder, and Beyond. Curr Obes Rep 2018;7:19-26.

[20] Kim J, Mutyala B, Agiovlasitis S, Fernhall B. Health behaviors and obesity among US children with attention deficit hyperactivity disorder by gender and medication use. Prev Med 2011.

[21] Nederkoorn C, Jansen E, Mulkens S, Jansen A. Impulsivity predicts treatment outcome in obese children. Behav Res Ther 2007;45:1071-5.

[22] Choudhry Z, Sengupta SM, Grizenko N, et al. Body weight and ADHD: examining the role of self-regulation. PloS One 2013;8:e55351.

[23] Krain AL, Castellanos FX. Brain development and ADHD. Clin Psychol Rev 2006;26:43344.

[24] Vieira de Melo BB, Trigueiro MJ, Rodrigues PP. Systematic overview of neuroanatomical differences in ADHD: Definitive evidence. Dev Neuropsychol 2018;43:52-68. 
[25] Perlov E, Philipsen A, Matthies S, et al. Spectroscopic findings in attentiondeficit/hyperactivity disorder: Review and meta-analysis. World J Biol Psychiatry 2009;10:355-65.

[26] Perlov E, van Elst LT, Buechert M, et al. H1-MR-spectroscopy of cerebellum in adult attention deficit/hyperactivity disorder. J Psychiatr Res 2010;44:938-43.

[27] Soliva JC, Moreno A, Fauquet J, et al. Cerebellar neurometabolite abnormalities in pediatric attention/deficit hyperactivity disorder: a proton MR spectroscopic study. Neurosci Lett 2010;470:60-4.

[28] Ben Amor L, Chantal S, Bairam A. Relationship between late preterm birth and expression of attention-deficit hyperactivity disorder in school-aged children: clinical, neuropsychological, and neurobiochemical outcomes. Res Rep Neonatol 2012:77.

[29] American Psychiatric Association. Diagnostic and statistical manual of mental disorders: DSM-IV-TR. American Psychiatric Pub; 2000.

[30] Wechsler D. WISC-IV: Wechsler Intelligence Scale for Children, 4th Edition Integrated: Technical and Interpretive Manual. Harcourt Brace and Company; 2004.

[31] Wechsler D. Wechsler Preschool and Primary Scale of Intelligence: WPPSI-III. PsychCorp.; 2002.

[32] Conners CK. Conners 3rd Edition: Manual. Toronto, Ontario, Canada: MHS; 2008.

[33] Gianarris WJ, Golden CJ, Greene L. The Conners' Parent Rating Scales: a critical review of the literature. Clin Psychol Rev 2001;21:1061-93.

[34] Conners CK. Conners' Continuous Performance Test (CPT II): Version 5 for Windows : Technical Guide and Software Manual. MHS; 2004.

[35] Davis C, Levitan RD, Smith M, et al. Associations among overeating, overweight, and attention deficit/hyperactivity disorder: A structural equation modelling approach. Eat Behav 2006;7:266-74.

[36] Thamotharan S, Lange K, Zale EL, et al. The role of impulsivity in pediatric obesity and weight status: A meta-analytic review. Clin Psychol Rev 2013;33:253-62.

[37] Van Egmond-Fröhlich AWA, Widhalm K, de Zwaan M. Association of symptoms of attention-deficit/hyperactivity disorder with childhood overweight adjusted for confounding parental variables. Int J Obes 2012;36:963-8.

[38] Tripp G, Schaughency EA, Clarke B. Parent and teacher rating scales in the evaluation of attention-deficit hyperactivity disorder: contribution to diagnosis and differential diagnosis in clinically referred children. J Dev Behav Pediatr JDBP 2006;27:209-18.

[39] Van den Berg L, Pieterse K, Malik JA, et al. Association between impulsivity, reward responsiveness and body mass index in children. Int J Obes 2005 2011;35:1301-7. 
[40] Rezazadeh SM, Wilding J, Cornish K. The relationship between measures of cognitive attention and behavioral ratings of attention in typically developing children. Child Neuropsychol J Norm Abnorm Dev Child Adolesc 2011;17:197-208.

[41] Rizzutti S, Sinnes EG, Scaramuzza LF, et al. Clinical and neuropsychological profile in a sample of children with attention deficit hyperactivity disorders. Arq Neuropsiquiatr 2008;66:821-7.

[42] Edwards MC, Gardner ES, Chelonis JJ, et al. Estimates of the validity and utility of the Conners' Continuous Performance Test in the assessment of inattentive and/or hyperactiveimpulsive behaviors in children. J Abnorm Child Psychol 2007;35:393-404.

[43] Egeland J, Johansen SN, Ueland T. Differentiating between ADHD sub-types on CCPT measures of sustained attention and vigilance. Scand J Psychol 2009;50:347-54.

[44] Husarova V, Ondrejka I, Tonhajzerova I. Potential pathomechanisms of ADHD based on neurometabolite changes. Neuro Endocrinol Lett 2010;31:438-45.

[45] Ben Amor L, Drolet C, Chantal S, et al. The Link Between Pregnancy-Related Complications and Changes in Brain Metabolites in Children with Attention Deficit Hyperactivity Disorder. EC Neurol 2016;3:307:316.

[46] Cortese S, Kelly C, Chabernaud C, et al. Toward Systems Neuroscience of ADHD: A Meta-Analysis of 55 fMRI Studies. Am J Psychiatry 2012;169:1038-55.

[47] Diamond A. Executive Functions. Annu Rev Psychol 2013;64:135-68.

[48] Courvoisie H, Hooper SR, Fine C, et al. Neurometabolic functioning and neuropsychological correlates in children with ADHD-H: preliminary findings. $J$ Neuropsychiatry Clin Neurosci 2004;16:63-9.

\section{Tableaux}

Tableau 1. Caractéristiques cliniques et démographiques des participants

Tableau 2. Caractéristiques cliniques mesurées par les échelles de Conners

Légende : ${ }^{1}$ Des tests bilatéraux ont été utilisés pour toutes les variables, sauf pour l'échelle d'impulsivité *Valeur statistiquement significative

Tableau 3 ; Caractéristiques neuropsychologiques mesurées par le CPT-II

Légende : ${ }^{1}$ Des tests bilatéraux ont été utilisés pour toutes les variables, sauf pour l'échelle d'impulsivité *Valeur statistiquement significative

Tableau 4. Caractéristiques neurobiochimiques mesurées par la SRM-1H

Légende GPC: Glycérophosphocholine ; PCh: Phosphocholine; Cr: Créatinine; Glu: Glutamate ; Gln : Glutamine; mI: Myoinositol; NAA: N-acétylaspartate; NAAG: N-acétylaspartylglutamate *Valeur statistiquement significative 
Tableau 1. Caractéristiques cliniques et démographiques des participants

\begin{tabular}{|c|c|c|c|c|}
\hline \multicolumn{2}{|c|}{$(n=20$ participants $)$} & \multirow{2}{*}{$\begin{array}{c}\text { TDAH-SP } \\
9 / 1\end{array}$} & \multirow{2}{*}{$\begin{array}{c}\text { TDAH-PN } \\
9 / 1\end{array}$} & \multirow{2}{*}{$\begin{array}{c}\text { Échantillon total } \\
18 / 2\end{array}$} \\
\hline Sexe ratio $(H / F)$ & & & & \\
\hline Âge moyen en années (écart-type) & & $6,84(1,27)$ & $7,32(1,10)$ & $7,08(1,18)$ \\
\hline \multirow{6}{*}{ Revenu familial en dollars canadiens } & $<20000$ & 1 & 0 & 1 \\
\hline & 20000 à 39999 & 1 & 2 & 3 \\
\hline & 40000 à 59999 & 1 & 2 & 3 \\
\hline & 60000 à 80000 & 3 & 1 & 4 \\
\hline & $>80000$ & 3 & 2 & 5 \\
\hline & Inconnu & 1 & 3 & 4 \\
\hline \multirow{4}{*}{ Indice de Masse Corporelle en percentile } & $>15-\leq 50^{\mathrm{e}}$ & - & 6 & - \\
\hline & $>50-\leq 85^{\mathrm{e}}$ & - & 4 & - \\
\hline & $>85-\leq 97^{\mathrm{e}}$ (surpoids) & 7 & - & - \\
\hline & $>97^{\mathrm{e}}($ obésité $)$ & 3 & - & - \\
\hline \multirow{3}{*}{ Type de TDAH prédominant } & Inattentif & 1 & 4 & 5 \\
\hline & Hyperactif/Impulsif & 3 & 0 & 3 \\
\hline & Mixte & 6 & 6 & 12 \\
\hline \multirow{3}{*}{ Comorbidités psychiatriques } & Difficultés d'apprentissage & 0 & 1 & 1 \\
\hline & $\begin{array}{c}\text { Trouble oppositionnel avec } \\
\text { provocation }\end{array}$ & 3 & 2 & 5 \\
\hline & Trouble anxieux & 0 & 1 & 1 \\
\hline
\end{tabular}


Tableau 2. Caractéristiques cliniques mesurées par les échelles de Conners

\begin{tabular}{|c|c|c|c|c|c|}
\hline & & \multicolumn{2}{|c|}{ Moyenne (Ecart-type) } & \multirow{2}{*}{$\begin{array}{c}\text { Test de Wilcoxon des } \\
\text { rangs signés }\end{array}$} & \multirow{2}{*}{ Valeur $\mathbf{p}^{1}$} \\
\hline & & TDAH-SP & TDAH-PN & & \\
\hline \multicolumn{2}{|c|}{ Nombre de sujets $(\mathrm{n}=18)$} & 9 & 9 & & \\
\hline \multirow{4}{*}{$\begin{array}{l}\text { CPRS-R } \\
\text { (parents) }\end{array}$} & Variable inattention & $66,39(9,95)$ & $66,61(8,86)$ & $-1,50$ & 0,88 \\
\hline & Variable impulsivité & $66,72(10,71)$ & $64,28(9,44)$ & 4,00 & 0,31 \\
\hline & Variable CGI-Total & $64,33(9,88)$ & $63,17(10,93)$ & 3,50 & 0,66 \\
\hline & Variable DSM-Total & $67,67(9,29)$ & $66,50(7,91)$ & 2,50 & 0,82 \\
\hline \multirow{4}{*}{$\begin{array}{c}\text { CTRS-R } \\
\text { (professeurs) }\end{array}$} & Variable inattention & $65,67(13,56)$ & $64,00(7,78)$ & 6,50 & 0,48 \\
\hline & Variable impulsivité & $67,44(10,21)$ & $56,67(12,03)$ & 13,50 & $\mathbf{0 , 0 3 *}$ \\
\hline & Variable CGI-Total & $66,33(6,63)$ & $58,00(7,78)$ & 22,50 & $<0,01 *$ \\
\hline & Variable DSM-Total & $65,33(14,07)$ & $61,00(5,45)$ & 9,00 & 0,32 \\
\hline
\end{tabular}

${ }^{1}$ Des tests bilatéraux ont été utilisés pour toutes les variables, sauf pour l'échelle d'impulsivité *Valeur statistiquement significative 
Tableau 3. Caractéristiques neuropsychologiques mesurées par le CPT-II

\begin{tabular}{|c|c|c|c|c|}
\hline & Moyenı & Ecart-type) & Test de Wilcoxon des & Volour $n$ \\
\hline & TDAH-SP & TDAH-PN & rangs signés & Valeur p \\
\hline Nombre de sujets $(\mathrm{n}=18)$ & 9 & 9 & & \\
\hline Omissions & $53,94(11,33)$ & $72,89(38,77)$ & $-12,50$ & 0,16 \\
\hline Commissions & $53,13(6,12)$ & $54,02(8,21)$ & $-2,50$ & 0,41 \\
\hline Temps de réaction & $49,53(11,60)$ & $54,23(8,23)$ & $-7,50$ & 0,43 \\
\hline Erreur standard du temps de réaction & $56,75(10,54)$ & $63,24(9,17)$ & $-13,50$ & 0,13 \\
\hline Variabilité & $56,87(10,06)$ & $63,51(7,75)$ & $-16,50$ & 0,05* \\
\hline Persévérations & $56,20(9,64)$ & $79,04(35,11)$ & $-18,50$ & $\mathbf{0 , 0 3 *}$ \\
\hline
\end{tabular}

${ }^{1}$ Des tests bilatéraux ont été utilisés pour toutes les variables, sauf pour les commissions *Valeur statistiquement significative 
Tableau 4. Caractéristiques neurobiochimiques mesurées par la SRM-1H

\begin{tabular}{|c|c|c|c|c|c|}
\hline \multicolumn{2}{|c|}{ Nombre de sujets $(\mathrm{n}=12)$} & $\begin{array}{l}\text { Moyenne des différences } \\
\text { dans la paire }\end{array}$ & Écart type & $\begin{array}{c}\text { Statistique du test de } \\
\text { Wilcoxon des rangs signés }\end{array}$ & Valeur $\mathrm{p}$ \\
\hline \multirow{4}{*}{ Préfrontal droit } & $\mathrm{GPC}+\mathrm{PCh} / \mathrm{Cr}$ & $\mathbf{0 , 0 3}$ & $\mathbf{0 , 0 3}$ & 10,50 & $0,03 *$ \\
\hline & $\mathrm{Glu}+\mathrm{Gln} / \mathrm{Cr}$ & $-0,03$ & 0,48 & 1,50 & 0,81 \\
\hline & $\mathrm{mI} / \mathrm{Cr}$ & 0,05 & 0,16 & 4,50 & 0,44 \\
\hline & $\mathrm{NAA}+\mathrm{NAAG} / \mathrm{Cr}$ & 0,01 & 0,12 & 1,50 & 0,84 \\
\hline \multirow{4}{*}{ Préfrontal gauche } & $\mathrm{GPC}+\mathrm{PCh} / \mathrm{Cr}$ & $<-0,01$ & 0,04 & 0,50 & 1,00 \\
\hline & $\mathrm{Glu}+\mathrm{G} \ln / \mathrm{Cr}$ & $-0,19$ & 0,60 & $-0,50$ & 1,00 \\
\hline & $\mathrm{mI} / \mathrm{Cr}$ & $-0,04$ & 0,09 & $-3,50$ & 0,56 \\
\hline & $\mathrm{NAA}+\mathrm{NAAG} / \mathrm{Cr}$ & $-0,03$ & 0,21 & $-2,50$ & 0,69 \\
\hline \multirow{4}{*}{ Striatal droit } & $\mathrm{GPC}+\mathrm{PCh} / \mathrm{Cr}$ & 0,04 & 0,07 & 6,50 & 0,22 \\
\hline & $\mathrm{Glu}+\mathrm{Gln} / \mathrm{Cr}$ & 0,10 & 0,29 & 4,50 & 0,44 \\
\hline & $\mathrm{mI} / \mathrm{Cr}$ & 0,12 & 0,15 & 8,50 & 0,09 \\
\hline & $\mathrm{NAA}+\mathrm{NAAG} / \mathrm{Cr}$ & 0,03 & 0,11 & 3,50 & 0,56 \\
\hline \multirow{4}{*}{ Striatal gauche } & $\mathrm{GPC}+\mathrm{PCh} / \mathrm{Cr}$ & 0,04 & 0,05 & 8,50 & 0,09 \\
\hline & $\mathrm{Glu}+\mathrm{Gln} / \mathrm{Cr}$ & 0,31 & 0,70 & 5,50 & 0,31 \\
\hline & $\mathrm{mI} / \mathrm{Cr}$ & $-0,86$ & 1,98 & $-9,50$ & 0,06 \\
\hline & $\mathrm{NAA}+\mathrm{NAAG} / \mathrm{Cr}$ & $-0,07$ & 0,34 & $-0,50$ & 1,00 \\
\hline \multirow{4}{*}{ Cervelet gauche } & $\mathrm{GPC}+\mathrm{PCh} / \mathrm{Cr}$ & 0,04 & 0,04 & 8,50 & 0,09 \\
\hline & $\mathrm{Glu}+\mathrm{Gln} / \mathrm{Cr}$ & $-0,22$ & 1,36 & $-2,50$ & 0,69 \\
\hline & $\mathrm{mI} / \mathrm{Cr}$ & 0,08 & 0,15 & 5,50 & 0,31 \\
\hline & $\mathrm{NAA}+\mathrm{NAAG} / \mathrm{Cr}$ & $-0,21$ & 0,43 & $-6,50$ & 0,22 \\
\hline
\end{tabular}

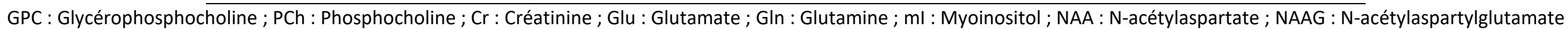

*Valeur statistiquement significative 